\title{
Editorial What the future holds for the Netherlands Journal of Geosciences
}

\author{
Johan ten Veen ${ }^{1, *, \dagger}$
}

1 TNO-Geological Survey of the Netherlands, Princetonlaan 6, 3584 СB Utrecht, the Netherlands

* Corresponding author. Email: johan.tenveen@tno.nl

$\dagger$ Editor-in-chief, also on behalf of the editors Geert-Jan Vis, Rick Donselaar, Fred Beekman, Ronald van Balen and Hanneke Verweij

My first year as Editor-in-Chief of the Netherlands Journal of Geosciences / Geologie en Mijnbouw turned out to be a steep learning curve which showed me all the facets this task brings. It has been a productive year, though. A total of 49 papers were published in three regular and two special issues. The special issues, on the Meuse Valley and on Seismicity of the Groningen Gas Field, were well received and contain papers authored by members of academic and applied institutions, including universities, consultants, advisory institutions, provinces, energy operators and regulatory authorities. This once again proves that thematic issues are a great strength of the Journal and it is good to notice that the Journal has become a medium for a broad geoscientific research community.

Your loyalty as an author, referee, editor or editorial board member is very important to us.

\section{Themed issues}

Without all the work of contributors, guest editors, associate editors and reviewers, 2017 would not have been such a great year; their efforts are highly appreciated. We will continue to organize and publish special themed issues that will be globally relevant and of national interest to the Netherlands and the neighbouring regions. In September a special issue will appear that is devoted to research on sea-level rise, subsidence and sedimentation in the Dutch Wadden Sea. It is really pleasing to see that initiatives for special issues are piling up and in the coming years you may expect at least two a year.

By continuing on the same path of success the Journal's future is bright.

\section{Scope and article types}

The Journal relies heavily on regular contributions and needs ambassadors who will promote the publication of articles on any geoscientific topic that complies with the scope of the Journal. The Journal publishes new and significant research in geosciences with a regional focus on the Netherlands, the North Sea region and the adjacent area. Unsurprisingly, given the geographical scope aspect, three-quarters of submissions are from the Netherlands. Whilst the geographical authorship of the journal is currently very narrow, we aim to publish on topics that are relevant to a wide audience globally. For instance, publications that describe analogues that pertain to the region are very welcome. Therefore we encourage all authors to submit to the Journal and, in case of doubt, inquire with the editors.

The Journal not only publishes regular papers, but also handles Geo(im)pulse and Geoperspective papers. The Geoimpulse papers are intended to be published rapidly, and should contain timely information. They will not exceed four printed pages and will contain at most one or two figures/tables. Geoperspective papers are short (max. four pages) contributions that provide a geoscientific viewpoint on societal issues.

\section{Our front cover}

As you may have noticed, the layout of the journal has undergone some changes, the most important of which is the change of the cover photo. A competition was held, and the new photo was selected for its geological content and because it so nicely fits the layout. It was taken and provided by Jeroen M. Peters and shows the tidal channels and 'English Corner' sand flats situated in between the Wadden islands of Vlieland and Terschelling.

\section{Transition to open access and online-only}

An even bigger change will occur in 2019 when the Netherlands Journal of Geosciences / Geologie en Mijnbouw will become fully gold open access (OA) and online-only. Whereas up until now it was possible to make any individual paper $0 \mathrm{~A}$, the $N J G$ board has proposed that the time is right to go completely gold OA. An 
official statement will be published shortly in KNGMG's (the Royal Geological and Mining Society of the Netherlands') Geo.Brief and on the news page of Cambridge University Press.

'Transitioning from a pay-walled subscription model to an online-only open access model will be hugely beneficial to the Journal. It will maintain the Journal's competitive position with its community and at the same time align better with the Dutch librarians' and EU Horizon 2020 mission to make research data more accessible to the readers,' says Dr Kathleen Too, Publisher for STM Journals at Cambridge University Press.
The new $0 \mathrm{~A}$ and online-only model proposed for the Journal will potentially increase the sustainability, impact and outreach of the Journal. With the help of loyal authors and referees, we are confident that the Journal will keep receiving a healthy number of submissions. The Editors and I will ensure a high-quality peer review process, fast publication speeds and maintain the high level of citations.

We hope that you will remain loyal readers and contributors to the Journal and look forward to receiving your new manuscripts. 